\title{
Absorbable sutures for the achievement of stable osteosynthesis in surgery for craniosynostosis
}

\author{
Frantisek Horn ${ }^{1}$, Evangelos Kilipiris ${ }^{2}$, Jennifer Pinzon ${ }^{3}$, Ioannis Tsolakis ${ }^{4}$, \\ Michal Kabat ${ }^{1}$, Michal Petrik ${ }^{1}$, Dana Kuniakova ${ }^{1}$ \\ ${ }^{1}$ Department of Paediatric Surgery, Division of Paediatric Neurosurgery, Medical Faculty of Comenius University in Bratislava \\ and National Institute of Children's Diseases, Slovakia \\ ${ }^{2}$ Department of Oral and Maxillofacial Surgery, Medical Faculty of Comenius University in Bratislava and University Hospital, Slovakia \\ ${ }^{3}$ Department of Oral and Maxillofacial Surgery, National University of Colombia and University Hospital of Bogota, Colombia \\ ${ }^{4}$ Department of Experimental Surgery and Surgical Research, School of Medicine, National and Kapodistrian University of Athens, Greece
}

\begin{abstract}
Aim of the study. The goal of the present study was to analyse the exclusive use of absorbable suture material (Vicryl) in the fixation of transposed bone segments in cranial vault reshaping without modification of the osteotomy design.

Clinical rationale for the study. In the surgical correction of craniosynostosis, bone fixation using osteosynthesis is a key step. Absorbable osteosynthesis is a widespread tool in cranial vault remodelling, but only a limited number of studies have described the use of absorbable sutures in the treatment of patients with craniosynostosis.

Materials and methods. In 72 children with various types of craniosynostosis, up to 24 months of age, osteosynthesis was conducted exclusively with Vicryl sutures. All patients were evaluated for the stability of postoperative results, and foreign body reactions were examined as part of the routine clinical and radiological follow-up ranging from 1 to 36 months.

Results. All examined children exhibited stable postoperative conditions with immediate stability of all remodelled cranial vaults. 2D and 3D radiological examinations demonstrated good bony union in all cases. Significant foreign body reactions were not observed and bone healing was noted at all sites.

Conclusion and clinical implications. The exclusive application of absorbable suture material enables stable and cost-effective osteosynthesis in craniofacial surgery without altering the osteotomy design.

Key words: craniosynostosis, craniofacial surgery, osteosynthesis, absorbable sutures, cranial vault reconstruction (Neurol Neurochir Pol 2019; 53 (2): 150-155)
\end{abstract}

\section{Introduction}

Craniosynostosis, the premature fusion of one or more cranial sutures, occurs in 1:2,000-2,500 live births, causing typical head shapes depending on the affected suture [1]. The management of craniosynostosis is multidisciplinary but the treatment is principally surgical. The surgical treatment of craniosynostosis consists of osteotomy, bone repositioning and fixation of the affected region using osteosynthesis [2].
There are many different techniques available to correct the deformity.

However, there continues to be a debate over the optimal procedure, timing of surgery and use of osteosynthesis material [3]. Stable osteosynthesis is dependent on immobile fixation of the communicating osteotomy edges and plays a crucial role in the successful outcome of the surgical procedure [4].

Historically, various techniques have been used for rigid fixation, each with different characteristics and properties.

Address for correspondence: Frantisek Horn, Department of Paediatric Surgery, Division of Paediatric Neurosurgery, Medical Faculty of Comenius University in Bratislava and National Institute of Children's Diseases, Slovakia,e-mail: frantisek.horn@gmail.com 
Simple metallic wire ligatures and metallic plates and screws were among the first materials to be implemented in osteosynthesis [5]. These enabled relatively stable as well as cost-effective fixation of the translocated bone segments [6]. The most common problems encountered when using this method include unwieldy handling, temperature-induced pain, risk of intracranial migration into the growing skull as a result of transposition of the osteosynthesis materials, danger of an injury to the overlying scalp, as well as the disadvantage of removing the material in a second surgical procedure. Recent developments in osteosynthesis materials include titanium microplates which enable simple, well defined and stable fixation [7]. Artifacts in radiological examinations and potential transosseous migration appeared as disadvantages from the use of these non-absorbable plates [8]. These problems seemed to be solved at the end of 1990s with the introduction of biodegradable absorbable osteosynthesis plates and screws composed of absorbable polymers [9]. Their use has been well documented over the past 15-20 years in the craniofacial literature. However, hydrolytic foreign body reaction, loss of tensile strength, less stabilisation and more difficult handling have been reported with the implementation of this method [10]. In addition, reports have shown that the use of absorbable plates increases the operation time due to the extra need for tapping the screw hole and the risk of screw fracture, when the screws are not applied accurately in an orthograde direction [11]. Similarly, published complications associated with resorbable materials are soft-tissue swelling, osteolysis and sterile fistulas, as well as the problem of palpability of the implanted plate resulting in a significant bulge preceding the complete degradation [12]. Studies have shown that the thickness of the plates increases by up to $300 \%$ during the degradation process [13].

A promising approach in the field of biodegradable osteosynthesis materials has been employed in our unit for up to 10 years. This simple, stable and cost-effective osteosynthesis method is offered through the correct fixation of displaced bone segments with absorbable sutures. In our department, absorbable sutures are used in the majority of cases to fixate transposed bone segments. Specifically, Vicryl sutures (Ethicon, Slovakia) are implemented in the treatment of craniosynostosis in children up to 2 years of age. Treatment of children over 2 years of age is carried out with polydioxanone sutures
(PDS, Ethicon, Slovakia) in order to exploit the extended time of resorption or with resorbable screws and plates.

\section{Clinical rationale for the study}

The current study was performed in children up to 2 years of age undergoing cranial vault reconstruction where the fixation of bone segments was performed exclusively with absorbable Vicryl sutures. The surgical procedure remained consistent with that of resorbable plate osteosynthesis. The purpose of the present paper was to accurately investigate and evaluate the overall efficacy of absorbable sutures in our series of craniosynostosis patients.

\section{Materials and Methods}

The current study was based on a retrospective design using a standard measurement protocol, examined and approved by the institutional Ethical Committee. The study was carried out according to the Declaration of Helsinki and written informed consent was obtained from the parents. A total of 72 children ( 39 male, 33 female) with premature closure of at least one cranial suture who were treated with primary cranial vault reconstruction were included in our series. The operations were performed between January 2008 and December 2017.

All cases were carried out by a single craniofacial-paediatric neurosurgical team, and rotating anaesthesiologists and paediatric intensive care specialists assigned to the team. Patient medical records were used to assess the length of surgery, estimated blood loss, average length of hospital stay, and interdisciplinary postoperative follow-up.

58 children with nonsyndromic single suture synostosis were investigated. Moreover, eight children presented with nonsyndromic complex craniosynostosis, and six with a premature suture closure associated with a syndrome (two children diagnosed with Apert syndrome, three with Crouzon syndrome, and one with Pfeiffer syndrome) (Tab. 1).

The average age at the time of surgery was 7.8 months, with a range between 3.5 and 21 months, depending on the involved suture or the patient's individual situation. Postoperatively, patients remained in the paediatric intensive care unit and were transferred to the paediatric neurosurgery ward thereafter.

Table 1. Distribution of involved sutures with summary of patients' demographic data

\begin{tabular}{|c|c|c|c|c|c|c|}
\hline & $\begin{array}{c}\text { Sagittal } \\
\text { Synostosis }\end{array}$ & $\begin{array}{c}\text { Metopic } \\
\text { Synostosis }\end{array}$ & $\begin{array}{l}\text { Unicornal } \\
\text { Synostosis }\end{array}$ & $\begin{array}{l}\text { Bicoronal } \\
\text { Synostosis }\end{array}$ & $\begin{array}{l}\text { Multisutural } \\
\text { Synostosis }\end{array}$ & $\begin{array}{l}\text { Syndromic } \\
\text { Synostosis }\end{array}$ \\
\hline Children & 36 & 16 & 2 & 4 & 8 & 6 \\
\hline Mean age at surgery time & 7.4 & 9.8 & 8 & 8.5 & 7.8 & 5.1 \\
\hline Mean postoperative hospital stay [days] & 5.7 & 7.8 & 4.5 & 6.8 & 5.3 & 8.2 \\
\hline Mean follow-up time [months] & 24.4 & 29.4 & 22 & 21.3 & 29.4 & 30.4 \\
\hline Complications & 0 & 1 & 0 & 1 & 1 & 0 \\
\hline
\end{tabular}


The average hospital stay was 6.4 days after surgery. The surgical procedure of choice was single stage open transcranial vault remodelling with barrel-staving osteotomies and orbital bandeau advancement as required for existing fronto-orbital dysmorphology. Absorbable sutures were applied to achieve a stable fixation of the transpositioned bone segments through the implementation of absorbable Vicryl sutures, sizes 2-0, 3-0 and 4-0 (Ethicon, Slovakia).

The brain remembers its normal shape. After osteotomy, after the fixation of the bone stenosis was abolished, the brain returned to its normal position. The aim of the stitches was to keep the bones in their new position and to create a normal cranial vault curve during healing. The sutures were applied through the burr-holes. The number of stitches used was as low as possible, usually two on each piece of bone to keep them in their normal position and to fix them with their neighbours.

Follow-up examinations occurred as part of the existing clinical routine protocol. Clinical evaluation was performed by the authors in consultation with a paediatric neurosurgeon and attending neuropaediatrician. Consistency of intraoperatively achieved stability was assessed through subjective inspection and palpation. Additionally, children were examined for possible signs of foreign body reaction specifically manifested through local redness, rejection or systemic reaction. In the postoperative period, routine $2 \mathrm{D}$ skull $\mathrm{X}$-rays on a regular basis and 3D CT one year after surgery were conducted, beside standardised anthropometric measurements.

\section{Results}

Of the 72 patients meeting the selection criteria of this study, 58 presented with single suture synostosis, and 14 with multiple suture involvement. From those with monosutural synostosis, 36 were discovered to have premature closure of the sagittal suture. Surgical treatment was carried out in one operative setting through open cranial vault reconstruction with or without superior orbital rim reshaping depending on the part of the sagittal suture fusion (i.e. anterior half, posterior half, or both) at the mean age of 7.4 months (Fig. 1). 16 children demonstrated a premature closure of the metopic suture. Surgical approach of choice involved metopic suture release, simultaneous rim advancements, and lateral widening via frontal bone advancement at a mean age of 9.8 months. The two children enrolled in the study with premature unilateral coronal synostosis had a median age of 8 months at the time of operation, and the surgical therapy included simultaneous frontal bone and bilateral orbital rim advancement. Four children with premature bilateral coronal suture synostosis were involved in the study. Fronto-orbital advancement was performed to treat this group as well, at a mean age of 8.5 months at the time of operation.

Eight children with multiple suture synostosis presented to our clinic. The surgical correction of this group of patients was achieved with combined approaches of open cranial vault

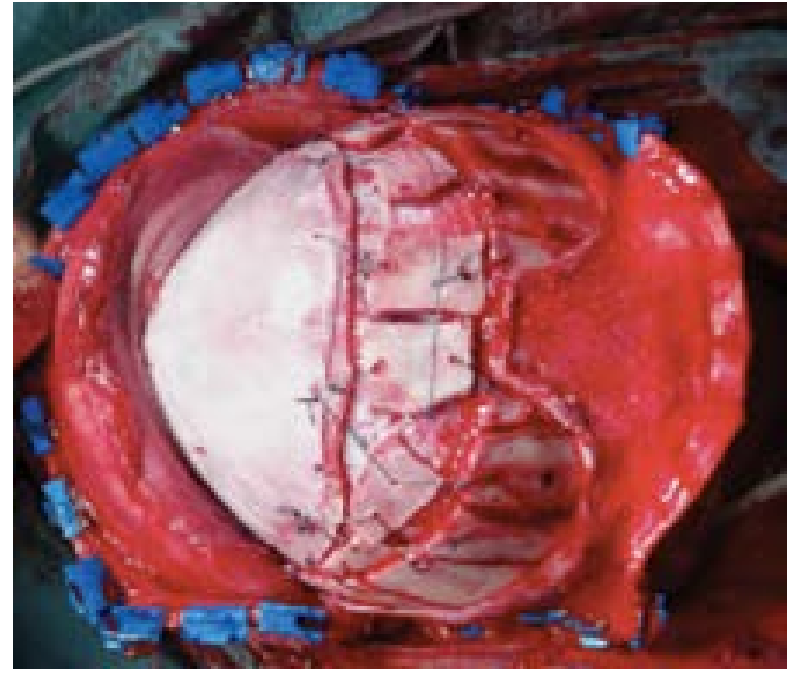

Figure 1. Intraoperatively, supine patient position after stable fixation of bone segments using Vicryl sutures in a child with sagittal suture synostosis and a normal supraorbital region. Superior view

reshaping (Fig. 2). The average age at surgery was 7.8 months. In addition to the previously described non-syndromic cases, six children with syndromic craniosynostosis who fulfilled the inclusion criteria were included in this analysis. Surgical treatment consisted of complete open skull remodelling with bone transposition, and the average age at surgery was 5.1 months.

The mean length of postoperative hospitalisation stay of patients with sagittal suture craniosynostosis was 5.7 days. Children with metopic suture involvement were discharged from the clinic an average of 7.8 days after surgery. One patient underwent a prolonged hospital course secondary to wound infection, which was treated with oral cephalexin, and was released after four days. The children in the unicoronal synostosis subgroup left the clinic at an average 4.5 days following surgery. All children after bicoronal synostosis correction left hospital at an average of 6.8 days postoperatively, except for one case who developed a subgaleal hygroma requiring puncture. Discharge of the children with multiple suture craniosynostosis from our department took place 5.3 days after reconstruction. Intraoperatively, one patient from this subgroup, with fusion of the metopic and sagittal sutures displayed sagittal sinus bleed immediately after performing the osteotomies for the orbital bandeau and creation of frontal and parietal craniotomies. Hypotension and brief asystole followed. The child was stabilised through successful local haemostatic techniques and adequate volume resuscitation control of haemorrhage. The patient tolerated the operation well and was observed for an additional seven days for any signs of intracranial bleeding or CSF leakage. Finally, the length of hospitalisation following primary surgery in the group of patients with syndromic craniosynostosis was an average of 8.2 days. No surgically related complications were encountered. 


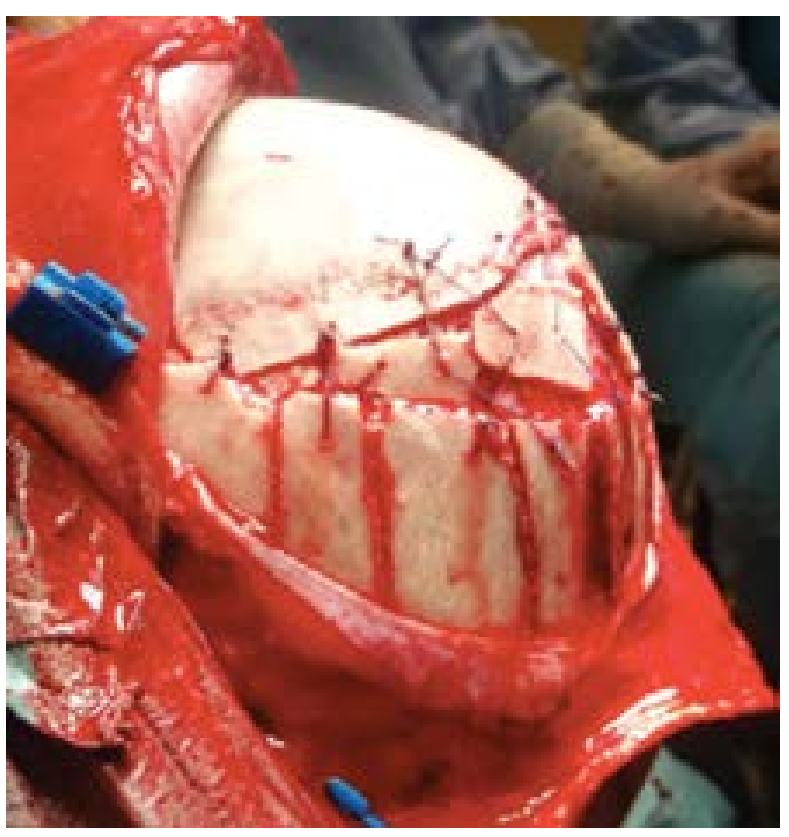

Figure 2. Intraoperatively, final reshaping of bone flaps performed with Vicryl sutures of a 9 month-old child with metopic and anterior half of sagittal suture fusion. The metopic suture fusion did not affect the orbital rims. Lateral view

Wound healing was uneventful during the inpatient period. During the following outpatient clinical course, irritation-free healing of the operative wound was monitored in all patients and all remodelled cranial vaults were stable on clinical palpation. Clinical signs of inflammation or infection in the area of previous surgery were not seen in the complete follow-up period. No signs of foreign body reaction to the resorbable material were observed. Clinical follow-up was carried out for an average of 26.2 months, ranging from 1 to 36 months.

In addition, postoperative cranial imaging including radiographic examination with skull X-ray and CT was performed for an average of 12 months. The follow-up radiographic imaging allowed the evaluation of the degree of ossification as well as the stability of the intraoperative bone relocation. Relevant complications concerning osteosynthesis of the transposed bone segments were not observed, and further interventions were not necessary. The radiographs demonstrated stable postoperative results with a firm bony union and a maintained degree of displacement in all cases. Postoperative dislocations resulting from a loosening or tear of the applied suture material were not observed.

Perioperative and postoperative outcomes were satisfactory in all patients. Parents reported no behavioural or psychomotor abnormalities after surgery. The results on functional, morphologic and aesthetic levels were good. In all cases, the cosmetic outcome was acceptable to the parents during the last follow-up appointment.

\section{Discussion}

In the surgical treatment of craniosynostosis, the achievement of stable fixation of transposed bone segments is the foundation of successful therapy. Close, firm and stable communication of the osteotomy edges will lead to direct healing of the bone [15]. Previously published studies based on the use of non-absorbable osteosynthesis material showed unacceptably high rates of complications and morbidity including displacement of the material into the child's growing skull, as well as the need for a second operation for removal of this material.

Over time, the method of osteosynthesis has evolved to witness the replacement of non-absorbable materials such as wire ligatures and titanium microplates with absorbable screws and plates. Most of them are based on resorbable miniplates and miniscrews. The majority of the systems available are derived from polymers and copolymers of glycolic acid, lactic acid and mixtures of D- and L-lactides. Although these resorbable osteosynthesis systems have advanced the technique of bony fixation in craniofacial surgery, some disadvantages have been described.

Published complications associated with resorbable materials include foreign body reactions, soft tissue swelling, reduced stability if the bone exhibits insufficient bone thickness, osteolysis and sterile fistulas [16]. In addition, fractures of absorbable plates have also been reported. Furthermore, during follow-up the number of palpable or visible plates, respectively, increases during the first months up to a maximum of 12 months [17]. It has been reported that the thickness of the plates increases by up to $300 \%$ during the degradation process. This initial swelling effect of the resorbable plates which results in a significant bulge preceding the complete degradation has been described by various authors [18].

Basic factors that an osteosynthesis material should fulfill include stability, high biocompatibility, fast and simple use as well as cost effectiveness. Furthermore, the current surgical technique itself should only be marginally changed through the implementation of osteosynthesis material. In addition, the young age of many treated craniosynostosis patients and the unique pattern of cranial vault growth influence the material selection [19].

In the current study, 72 children underwent open cranial vault remodelling with fixation of transposed bone segments performed exclusively with Vicryl sutures (Ethicon, Slovakia). In almost all cases, Vicryl 3-0 and 4-0 was used except for events of an increased tensile load, where Vicryl 2-0 was employed. Long-term follow-up examinations with clinical assessments showed stable osteosynthesis results in all cases. Moreover, foreign body reaction manifesting as local redness, inflammatory skin reaction, rejection or systemic response was not observed in our study group. 
The absence of these findings confirms the results of a recent clinical study of 129 patients described by Linz et al. where stable osteosynthesis was achieved absolutely with the use of Vicryl (Ethicon, Germany) suture material. This is in contrast to numerous studies in the literature which describe similar adverse reactions from the use of absorbable plates and screws [20]. According to another clinical study performed by Sanger et al., the complication rates from the use of Vicryl sutures for osteosynthesis were under 1\% [21]. This concept was further supported by Gonzalez et al. as they reported a consistent positive assessment with an absence of complications in a group of 37 patients. In our series, follow-up radiographic investigations were employed with 3DCT one year postoperatively and $2 \mathrm{D}$ skull $\mathrm{X}$-rays at regular intervals during the first 36 months, and then if indicated until the end of skull growth at the age of 12 years. The outcome in all cases supported the findings of stable bony union without dislocation of the transpositioned segments.

The Vicryl suture material fills the need for a smoother synthetic absorbable suture to facilitate ease of handling, smooth tie down and unsurpassed knot security. Knots are tied with precision and each must hold with proper tension. Adequate strength of the suture material will prevent suture breakage. Secure knots prevent knot slippage and reliable stability is achieved by implementation of stable sutures. According to the manufacturer, at two weeks post-implantation, approximately $75 \%$ of the original tensile strength of Vicryl suture remains. The breaking force begins to lessen over a period of 4-5 weeks. Absorption of Vicryl suture is essentially complete between 56 and 70 days.

It should be noted at this point that the use of Vicryl sutures is shown to reduce the cost in comparison to the high material cost for osteosynthesis with absorbable plates. This is in line with today's healthcare environment that requires hospitals to continue to maintain quality standards while lowering material costs to remain financially viable.

Clinical implications/Future directions Adequate positioning and fixation of the bone segments is a fundamental element in paediatric craniofacial surgery. The data collected and presented in this study has enabled the confirmation of a stable osteosynthesis, with the exclusive use of Vicryl sutures in the reconstruction of children with craniosynostosis up to two years of age. All patients showed a favourable outcome. From a long-term point of view, this series found that Vicryl sutures provide an effective, rigid, well-tolerated and economical alternative osteosynthesis method with satisfying results.

Funding This publication was prepared without any external sources of funding.

Conflict of interest The authors declare that they have no conflict of interest.

\section{References}

1. Striker MV, Raphael B, Mazzola R. Craniofacial malformations. 1st ed. Churchill Livingstone, Edinburgh 1990: chapter 7.

2. Whitaker LA, Bartlett SP, Schut L, et al. Craniosynostosis: an analysis of the timing, treatment, and complications in 164 consecutive patients. Plast Reconstr Surg. 1987; 80(2): 195-212, indexed in Pubmed: 3602170.

3. Tharanon W, Sinn DP, Hobar PC, et al. Surgical outcomes using bioabsorbable plating systems in pediatric craniofacial surgery. J Craniofac Surg. 1998; 9(5): 441-4; discussion 445, indexed in Pubmed: 9780913.

4. Barone CM, Jimenez DF. Special considerations in pediatric cranial fixation: a technical overview. J Craniomaxillofac Trauma. 1996; 2(4): 42-47, indexed in Pubmed: 11951449.

5. Yaremchuk MJ. Experimental studies addressing rigid fixation in craniofacial surgery. Clin Plast Surg. 1994; 21(4): 517-524, indexed in Pubmed: 7813152.

6. Fearon J. Rigid Fixation of the Calvaria in Craniosynostosis without Using "Rigid" Fixation. Plastic and Reconstructive Surgery. 2003; 111(1): 27-38, doi: 10.1097/01.prs.0000036261.99248.09.

7. Ohata K, Haque M, Tsuruno T, et al. Craniotomy repair with titanium miniplates. J Clin Neurosci. 1998; 5(1): 81-86, indexed in Pubmed: 18644295.

8. Goldberg DS, Bartlett S, Yu JC, et al. Critical review of microfixation in pediatric craniofacial surgery. J Craniofac Surg. 1995; 6(4): 301-7; discussion 308, indexed in Pubmed: 9020705.

9. Obwegeser JA. [Absorbable and bioconvertible osteosynthesis materials in maxillofacial surgery]. Mund Kiefer Gesichtschir. 1998; 2(6): 288-308, doi: 10.1007/s100060050077, indexed in Pubmed: 9880999.

10. Kumar CR, Sood S, Ham S. Complications of bioresorbable fixation systems in pediatric neurosurgery. Childs Nerv Syst. 2005; 21(3): 205-210, doi: 10.1007/s00381-004-0997-0, indexed in Pubmed: 15278386.

11. Losken A, Williams J, Burstein F, et al. Outcome Analysis for Correction of Single Suture Craniosynostosis Using Resorbable Fixation. Journal of Craniofacial Surgery. 2001; 12(5): 451-455, doi: 10.1097/00001665-200109000-00009.

12. Pietrzak WS, Verstynen ML, Sarver DR. Bioabsorbable fixation devices: status for the craniomaxillofacial surgeon. J Craniofac Surg. 1997; 8(2): 92-96, indexed in Pubmed: 10332273.

13. Eppley BL, Li M. Long-spanning resorbable plates in cranial vault reconstruction. J Craniofac Surg. 2003; 14(1): 89-91, indexed in Pubmed: 12544228.

14. Pagnoni M, Fadda MT, Spalice A, et al. Surgical timing of craniosynostosis: what to do and when. J Craniomaxillofac Surg. 2014; 42(5): 513-519, doi: 10.1016/j.jcms.2013.07.018, indexed in Pubmed: 24075801.

15. Sauerhammer TM, Seruya M, Basci D, et al. Endocortical plating of the bandeau during fronto-orbital advancement provides safe and effective osseous stabilization. J Craniofac Surg. 2014; 25(4): 1341-1345, doi: 10.1097/SCS.0000000000000810, indexed in Pubmed: 24902106.

16. Duke BJ, Mouchantat RA, Ketch LL, et al. Transcranial migration of microfixation plates and screws. Case report. Pediatr Neurosurg. 1996; 25(1): 31-4; discussion 35, doi: 10.1159/000121093, indexed in Pubmed: 9055332. 
17. Freudlsperger C, Castrillon-Oberndorfer G, Baechli H, et al. The value of ultrasound-assisted pinned resorbable osteosynthesis for cranial vault remodelling in craniosynostosis. J Craniomaxillofac Surg. 2014; 42(5): 503-507, doi: 10.1016/j.jcms.2013.07.016, indexed in Pubmed: 24011607.

18. Eckelt U, Nitsche M, Müller A, et al. Ultrasound aided pin fixation of biodegradable osteosynthetic materials in cranioplasty for infants with craniosynostosis. J Craniomaxillofac Surg. 2007; 35(4-5): 218-221, doi: 10.1016/j.jcms.2007.04.005, indexed in Pubmed: 17681775.
19. Kosaka M, Miyanohara T, Wada Y, et al. Intracranial migration of fixation wires following correction of craniosynostosis in an infant. J Craniomaxillofac Surg. 2003; 31(1): 15-19, indexed in Pubmed: 12553921.

20. Wood RJ, Petronio JA, Graupman PC, et al. New resorbable plate and screw system in pediatric craniofacial surgery. J Craniofac Surg. 2012; 23(3): 845-849, doi: 10.1097/SCS.0b013e31824dbed8, indexed in Pubmed: 22565906.

21. Montag ME, Morales L, Daane S. Bioabsorbables: their use in pediatric craniofacial surgery. J Craniofac Surg. 1997; 8(2): 100-102, indexed in Pubmed: 10332275. 